Vortragsabend der Akademie der Wissenschaften zu Göttingen im Niedersächsischen Landtag in Hannover am 21. November 2016

\title{
Dirk Schumann \\ Berlin ist nicht Weimar: Die Weimarer Republik und ihre politische Kultur
}

Philosophische Fakultät der Universität Göttingen

\section{Einleitung}

Lange Zeit war die Weimarer Republik eine ferne, zugleich aber auch präsente Vergangenheit. In der alten Bundesrepublik erschien sie als „negative Kontrastfolie“ und in Situationen besonderer innenpolitischer Spannung als Referenzpunkt für konkrete Warnungen, wenngleich mit einer seit den 1970er Jahren abnehmenden Intensität. ${ }^{1}$ Die Umbrüche der Jahre 1989/90 und der neue Globalisierungsschub haben den wahrgenommenen Abstand zu ihr dann zunächst deutlich vergrößert. Doch die neue politische Unübersichtlichkeit, die Erosion der Volksparteien und der Aufstieg einer neuen rechtspopulistischen Bewegung, deren Parolen an längst vergangen geglaubtes Gedankengut erinnern, sowie auch die Zunahme politisch motivierter Gewalt werfen die Frage auf, ob die Weimarer Jahre der Gegenwart vielleicht doch näher sind als lange angenommen. „Bonn ist nicht Weimar“ - dieser Satz des Schweizer Journalisten Fritz René Allemann ${ }^{2}$ war über Jahrzehnte hinweg die feste Überzeugung der politischen Beobachter in der Bundesrepublik. Gilt dies auch für die „Berliner Republik“, ist „Berlin“ in diesem Sinn also „nicht Weimar“? Die einfache Negation wäre eine zu schlichte Antwort, denn nicht nur die auf den ersten Blick ähnlichen Phänomene in der heutigen Politik lassen das Verhältnis beider Republiken deutlich komplexer erscheinen. Vor allem aber legt dies auch das von der Forschung mittlerweile gezeichnete Bild der Weimarer Republik nahe, in dem sich deren politische Entwicklung eben nicht allein als Katastrophengeschichte darstellt.

Die folgenden Überlegungen sollen zeigen, dass die Weimarer Republik auf der einen Seite tatsächlich eine ferne Vergangenheit ist und für eine markant andere Gesellschaft und Politik steht als die der Gegenwart. Deutlich werden soll auf der anderen Seite aber auch, dass die Weimarer Republik doch erheblich

1 Zur negativen Vorbildwirkung der Weimarer Republik in den Anfangsjahren der Bundesrepublik: Sebastian Ullrich, Der Weimar-Komplex. Das Scheitern der ersten deutschen Demokratie und die politische Kultur der frühen Bundesrepublik 1945-1959, Göttingen 2009, das Zitat S. 616. 2 Fritz René Allemann, Bonn ist nicht Weimar, Köln 1956. 
größere Erfolgschancen besaß, als dies lange behauptet wurde. Sie ist deshalb als eine zwar unter schwierigen Bedingungen operierende, zumindest bis zur Weltwirtschaftskrise ab 1929 aber grundsätzlich funktionsfähige Demokratie zu verstehen. Unter dieser Perspektive ist sie der Bundesrepublik der Gegenwart dann doch nicht ganz so unähnlich und insofern lassen sich doch gewisse Lehren aus ihr - oder genauer: aus ihrer politischen Kultur - ziehen. Solche Lehren können freilich nicht die Form ganz konkreter Handlungsanweisungen annehmen, die Geschichte nie bereithält, sondern sie bilden einen Beitrag zur stärkeren Sensibilisierung für Probleme der Gegenwart und für Strategien, mit ihnen umzugehen.

Im Mittelpunkt der folgenden Ausführungen stehen also Grundzüge und Wandel der politischen Kultur der Weimarer Republik und deren Beitrag zu einem besseren Verständnis der Geschichte der ersten deutschen Demokratie. Regionale Unterschiede, auf die der doppeldeutige Titel auch anspielt, können dabei nur gestreift werden. Nach knappen Bemerkungen zur Perspektive der historischen Forschung werden zunächst die mentalen Grundbefindlichkeiten der Weimarer Epoche behandelt, die gleichsam das Fundament ihrer politischen Kultur bildeten (II). Auf der Basis einer genaueren Erläuterung des Konzepts „politische Kultur“ (III) werden dann zuerst das Grundproblem ihrer Fragmentierung in der Weimarer Republik (IV) und anschließend die mittlerweile deutlicher erkennbaren Chancen ihrer Stabilisierung erörtert (V). Dem folgen knappere Überlegungen zu weiteren ihrer Belastungen, insbesondere zur politisch motivierten Gewalt (VI). Am Ende steht der Versuch, Bezüge zur Gegenwart herzustellen (VII).

\section{Die Weimarer Republik als Nachgeschichte großer Umwälzungen}

Lange Zeit haben verständlicherweise die Gründe für das Scheitern der Republik im Zentrum des wissenschaftlichen Interesses gestanden. So richtete sich die Perspektive auf die radikalen Kräfte an den Rändern des politischen Spektrums, auf Kommunismus und Nationalsozialismus, aber auch auf Mängel der Verfassung, Defizite der Parteien und verhängnisvolle Entscheidungen der führenden Politiker und Vertreter gesellschaftlicher Eliten. Von der - je nach Perspektive „überflüssigen“, „gescheiterten“ oder „steckengebliebenen“ Revolution 1918/19 schien, in einer von Kriegsfolgen belasteten Republik vermeintlich „ohne Republikaner“, ein kaum vermeidbarer Weg zur nationalsozialistischen Massenmobilisierung in der großen Wirtschaftskrise und zur NS-Machtübernahme zu führen. ${ }^{3}$

3 Zum Gang der Forschung zur Geschichte der Weimarer Republik und dem Wandel ihrer Deutungsansätze: Eberhard Kolb/Dirk Schumann, Die Weimarer Republik, München, 8. überarbeitete und erweiterte Aufl. 2013 (= Oldenbourg Grundriss der Geschichte, Bd. 16), S. 155ff. 
Diese vertraute Perspektive ist nicht gänzlich überholt, aber sie ist einseitig und unvollständig. Um zu verstehen, was die Menschen der Epoche umtrieb, ist es zunächst notwendig, sich von einer Fixierung auf die Geschichte der Weimarer Republik als Vorgeschichte der nationalsozialistischen Herrschaft zu lösen. Das ist sie zweifellos auch, und die Frage, warum es 1933 zur Machtübernahme kam - die zunächst eine Machtübergabe war - diese Frage bleibt wichtig, aber sie muss mit anderen Fragen verbunden werden und insoweit ein wenig an die Seite rücken. Die Weimarer Republik war nicht nur Vorgeschichte des Nationalsozialismus, sie war auch Nachgeschichte, Nachgeschichte nicht nur von Krieg und Revolution, sondern auch dessen, was man als Umwälzungen der „Moderne“ bezeichnen kann.

Damit sind die mentalen Grundbefindlichkeiten der Epoche angesprochen. Anzusetzen wäre etwa beim 1871 geborenen Friedrich Ebert und anderen nur wenig jüngeren Politikern wie Konrad Adenauer, Jahrgang 1876, oder Gustav Stresemann, Jahrgang 1878. Im Jahr der Reichsgründung lebten in Deutschland 41 Millionen Menschen - 1910, am Vorabend des Ersten Weltkriegs, waren es fast 65 Millionen. Die deutsche Bevölkerung hatte sich also in vierzig Jahren um mehr als die Hälfte vergrößert. Stark gewachsen waren dabei die Städte, gerade die großen: Hatten im Jahrzehnt der Reichsgründung nur fünf Prozent der Deutschen in einer Großstadt gewohnt, waren es 1914 bereits mehr als zwanzig Prozent. ${ }^{4}$ Schon diese dürren Zahlen lassen ahnen, wie dynamisch sich die deutsche Gesellschaft bis zum Vorabend des Ersten Weltkriegs veränderte. Es war fast zur Hälfte eine Gesellschaft der Jugend, der Unter-Zwanzigjährigen. Jung, zwischen 20 und 30, war auch die Mehrzahl der Binnenwanderer in die Städte, zudem waren sie vor allem männlich, ledig und sie waren Arbeiter. ${ }^{5}$ Schon dies deutet die Doppelgesichtigkeit, die innere Gespaltenheit der Wilhelminischen Gesellschaft an: Auf der einen Seite standen große Hoffnungen auf die Kraft der Jugend, auf der anderen tiefe Ängste vor Entwurzelung und Kontrollverlust. ${ }^{6}$

Weitere große, gleichfalls ambivalent empfundene Veränderungen sind $\mathrm{zu}$ nennen: Um 1900 war Deutschland seiner Wertschöpfung und seiner Beschäftigtenverteilung nach zum Industriestaat geworden - das Land, die ländliche Lebenswelt mit ihrer Enge, aber auch ihrer scheinbaren Geborgenheit trat in den Hintergrund. Die großen Städte kämpften mit Wohnungsproblemen, bildeten aber

\footnotetext{
4 Hans-Ulrich Wehler, Deutsche Gesellschaftsgeschichte. Dritter Band: Von der „Deutschen Doppelrevolution“ bis zum Beginn des Ersten Weltkriegs, 1849-1914, München 1995, S. 494, 512. 5 Ebd., S. 505; Thomas Nipperdey, Deutsche Geschichte 1866-1918. Erster Band: Arbeitswelt und Bürgergeist, München 1990, S. 10-20, 30 (mit ausführlicher Diskussion des markanten Rückgangs zunächst der Kinder- und Jugend- und dann der Säuglingssterblichkeit).

6 Nipperdey, Deutsche Geschichte (wie Anm. 5), S. 112-124.
} 
auch schon eine Reihe im besten Sinn moderner Einrichtungen aus: Schlacht- und Viehhöfe, Leihbibliotheken, Tierparks, erste Sportanlagen. Steigende Reallöhne und sinkende Arbeitszeiten erweiterten Schritt für Schritt die Möglichkeiten, an dem sich herausbildenden Freizeit- und Konsumangebot teilzuhaben. ${ }^{7}$ Tiefgreifend wandelte sich die Medienlandschaft, in der das Bild einen ungeahnten Aufstieg erlebte. Das alte Medium der Presse expandierte, konzentriert auf Unterhaltung und das aufgrund technischer Innovationen jetzt massenhaft einsetzbare Bild. So hatte die 1891 gegründete und neben dem Abonnement auch über den Straßenverkauf erhältliche Berliner Illustrirte Zeitung 1914 bereits eine Auflage von einer Million Exemplare. Auch das neue Medium des Films war bereits jetzt präsent und bescherte dem Kino seit 1906 einen regelrechten Gründungsboom, der selbst mittelgroße Städte wie Osnabrück und Oldenburg erfasste. ${ }^{8}$

Zweierlei wird damit deutlich: Erstens veränderten sich die deutsche Gesellschaft und die persönliche Lebenswelt, zumal die städtische, in den Jahrzehnten zwischen der Reichsgründung und dem Beginn des Ersten Weltkriegs, enorm. Gerade die in den 1870er und 1880er Jahren Geborenen erlebten diese Veränderungen bewusst mit und vor allem aus dieser Alterskohorte rekrutierten sich später die politische und die anderen Funktionseliten der Weimarer Republik. Zweitens war die moderne urbane Lebenswelt mit ihrer ausgeprägten Visualität schon vor 1914 in ihren wesentlichen Zügen ausgebildet. Die vielgerühmte ,Weimarer Kultur“ ist deshalb nicht ganz so neu, wie lange angenommen, in ihrer massenkulturellen und auch in ihrer hochkulturellen Variante. Also hängt die Weimarer Republik, was die Erfahrung von Umbrüchen und neuen kulturellen Impulsen angeht, an mehr alten, ins Kaiserreich weisenden Fäden, als dies zunächst scheinen mag.

Einschneidend ist dann ohne Zweifel die Erfahrung des Ersten Weltkriegs. Die massenhafte Gewalt, das massenhafte Töten und Sterben erschütterten tief Hoffnungen auf stetigen Fortschritt und wachsende Prosperität. Die Niederlage, die - je nach Standpunkt zu kurz greifende oder zu weit gehende - Revolution und die Inflation, vor allem deren letzte, im Krisenherbst 1923 kulminierende Phase der Hyperinflation, erzeugten weitere Ängste und Unsicherheiten. ${ }^{9}$

7 Wehler, Gesellschaftsgeschichte (wie Anm. 4), S. 523-543; Gerhard A. Ritter / Klaus Tenfelde, Arbeiter im Deutschen Kaiserreich 1871 bis 1914, Bonn 1992, S. 364-371, 491ff.

8 Corey Ross, Media and the Making of Modern Germany. Mass Communications, Society, and Politics from the Empire to the Third Reich, Oxford 2008, S. 23-44; Corinna Müller, Der frühe Film, das frühe Kino und seine Gegner und Befürworter, in: Kaspar Maase / Wolfgang Kaschuba (Hrsg.), Schund und Schönheit. Populäre Kultur um 1900, Köln 2001, S. 62-91, hier S. 63f.

9 Zur Erfahrung des Ersten Weltkriegs jetzt umfassend: Jörn Leonhard, Die Büchse der Pandora. Geschichte des Ersten Weltkriegs, München 2014; zur Erfahrung von Revolution und Inflation: 
Wie die vielfältigen Veränderungen erlebt wurden, hing auch von der materiellen Lage und der Milieuzugehörigkeit ab. Konservative Landbewohner, liberale Stadtbürger, sozialdemokratische Arbeiter und die nicht in einer spezifischen sozialen Schicht verortbaren Katholiken der Zentrumspartei nahmen die Welt unterschiedlich wahr, auch gemäß der Machtposition und der Erfolge der eigenen politischen Bewegung. Auch die Generationslagerung spielte eine Rolle: Die um das Reichsgründungsjahrzehnt Geborenen waren zu einer Zeit erwachsen geworden, um 1890, als das Kaiserreich in eine längere Phase robusten Wirtschaftswachstums eintrat. Die Sozialdemokraten unter ihnen hatten die Verfolgung unter dem Sozialistengesetz kaum mehr bewusst erlebt, hatten also weder intensive Leidenserfahrungen gemacht noch heldenhafte Kämpfe bestanden. So neigten sie, wie auch Politiker anderer Parteien aus ihrer Alterskohorte, zu einem eher pragmatisch-nüchternen Umgang mit politischer Macht. Das war bei den zwanzig Jahre jüngeren anders: Für sie bildete der Erste Weltkrieg die große einschneidende Erfahrung ihres Lebens. Sie tendierten zu größerer Radikalität und konnten während der Weimarer Jahre in den etablierten Parteien noch keine führenden Positionen erreichen, wohl aber in den neuen an den Rändern des politischen Spektrums. Der 1886 geborene Ernst Thälmann wäre hier zu nennen, seit Ende 1925 Vorsitzender der KPD, und der drei Jahre jüngere „Führer“ der NSDAP, der im selben Jahr seine Partei nach Putsch, Verbot und Haftzeit in Landsberg neu gründete. ${ }^{10}$

Diese Unterschiede in der politischen Grundhaltung zwischen den beiden Alterskohorten - mit Vorsicht als „Generationen“ $\mathrm{zu}$ bezeichnen ${ }^{11}$ - sind gewiss wichtig, aber sie sollen fundamentale Gemeinsamkeiten nicht verdecken. Beide und auch die noch etwas Älteren teilten eben, ungeachtet aller Unterschiede, die Erfahrung tiefgreifender Umbrüche hin zur „Moderne“, die teils Optimismus erzeugten, teils als krisenstiftend empfunden wurden und jedenfalls aktives Eingreifen verlangten. Eine Politik der ruhigen Hand, die Errungenes nur bewahren

Martin H. Geyer, Verkehrte Welt. Revolution, Inflation und Moderne: München 1914-1924, Göttingen 1998.

10 Bernd Braun, Die „Generation Ebert“, in: Klaus Schönhoven / Bernd Braun (Hrsg.), Generationen in der Arbeiterbewegung, München 2005, S. 69-86; Meik Woyke, Die „Generation Schumacher“, in: Schönhoven / Braun, Generationen, S. 87-106; Art. „Thälmann, Ernst“, in: Hermann Weber / Andreas Herbst, Deutsche Kommunisten. Biographisches Handbuch 1918 bis 1945, Berlin 22008, S. 925-928; Ian Kershaw, Hitler 1889-1936, Stuttgart 1998, bes. S. 338-348.

11 Zur Problematik der Zuordnungskategorie „Generation“ im hier untersuchten Zeitraum: Dirk Schumann, Youth Culture, Consumption, and Generational Dispositions in Twentieth-Century Germany. In: Hartmut Berghoff, Uffa Jensen, Christina Lubinski, Bernd Weisbrod (Hrsg.), History by Generations. Generational Dynamics in Modern History, Göttingen 2013, S. 125-146, bes. S. 131-137. 
wollte, konnte den Horizont der Weimarer Zeitgenossen nicht bestimmen. Und das lag nicht nur daran, dass nach Niederlage und Inflation und angesichts der alliierten Reparationsforderungen eine solche Politik nicht wirklich möglich war, sondern es hatte seinen Grund auch darin, dass die Vergangenheit kein klares Modell für ein solches Bewahren anbot.

So war die Weimarer Republik ein Staat und eine Gesellschaft ohne wirkliche Gegenwart und vom Bewusstsein geprägt, sich in einer Zeitenwende zu befinden. ${ }^{12}$ Nur wohin sollte diese Wende führen? Die Republikfeinde auf der Linken und der Rechten wünschten sich eine neue Revolution im jeweils eigenen Sinn und waren davon überzeugt, dass sie sehr bald schon eintreten könnte. Gerade auf der Rechten gab es über Schlagworte hinaus allerdings kein Modell für das dann erwünschte politische System. Die Befürworter der Republik wiederum kamen von recht unterschiedlichen weltanschaulichen Grundpositionen; sie handelten aus tiefer Überzeugung, aber auch aus vernunftgeleitetem Pragmatismus. Schon deshalb fiel es ihnen schwer zu bestimmen, was eine demokratische Republik im Einzelnen ausmachen sollte. ${ }^{13}$ Dem Zeitbewusstsein der Menschen in der Weimarer Republik haftete deshalb etwas Unruhiges an, etwas Tastendes, Suchendes, in der Politik wie in der „Kultur“ im weiteren Sinn. Hier kommen Politik und Kultur zusammen, in dieser Betonung des Handelns, Entscheidens, des Experimentierens, der Abkehr vom Gewohnten, was sich etwa in der Philosophie bei Karl Jaspers im Nachdenken über die existenziellen „Grenzsituationen“ manifestiert, oder - nicht nur beim zu Recht höchst umstrittenen Staatsrechtler Carl Schmitt - im Interesse am „Ausnahmezustand“. ${ }^{14}$

12 Martin H. Geyer, „Die Gleichzeitigkeit des Ungleichzeitigen“. Zeitsemantik und die Suche nach Gegenwart in der Weimarer Republik, in: Wolfgang Hardtwig (Hrsg.), Ordnungen in der Krise. Zur politischen Kulturgeschichte Deutschlands 1900-1933, München 2007, S. 165-187; Rüdiger Graf, Die Zukunft der Weimarer Republik. Krisen und Zukunftsaneignungen in Deutschland 1918-1933, München 2008.

13 Manfred Gangl / Gérard Raulet (Hrsg.), Intellektuellendiskurse in der Weimarer Republik. Zur politischen Kultur einer Gemengelage, Frankfurt 22007; Stefan Breuer, Anatomie der Konservativen Revolution, Darmstadt 1993; Kurt Sontheimer, Antidemokratisches Denken in der Weimarer Republik. Die politischen Ideen des deutschen Nationalismus zwischen 1918 und 1933, München 1978 (modifizierte Fassung der Studienausgabe von 1968); Christoph Gusy (Hrsg.), Demokratisches Denken in der Weimarer Republik, Baden-Baden 2000; Kathrin Groh, Demokratische Staatsrechtslehrer in der Weimarer Republik. Von der konstitutionellen Staatslehre zur Theorie des modernen demokratischen Verfassungsstaats, Tübingen 2010.

14 Martin H. Geyer, Grenzüberschreitungen: Vom Belagerungszustand zum Ausnahmezustand, in: Niels Werber / Stefan Kaufmann / Lars Koch (Hrsg.), Erster Weltkrieg. Kulturwissenschaftliches Handbuch, Stuttgart 2014, S. 341-384; Reinhard Mehring, Carl Schmitt. Aufstieg und Fall. Eine Biographie, München 2009, S. 76-129; Heiner Bielefeldt, Kampf und Entscheidung. Politischer Existenzialismus bei Carl Schmitt, Helmuth Plessner und Karl Jaspers, Würzburg 1994; 


\section{Zum Konzept der „politischen Kultur“}

Vor diesem Hintergrund ist die politische Kultur der Weimarer Republik zu diskutieren. Der nicht ganz scharf definierbare Begriff „politische Kultur“ meint die Einstellungen, Bilder und Symbole, samt begleitender Praktiken, die politisches Handeln grundsätzlich anleiten und auch sinnlich-emotional erfahrbar machen. ${ }^{15}$ Dazu gehören die Leitbegriffe der politischen Sprache, gerade solche, die lagerübergreifend benutzt, aber mit durchaus unterschiedlichen Inhalten gefüllt wurden. Ein Beispiel dafür ist der Begriff der „Volksgemeinschaft“, dessen prominente Verwendung während der NS-Herrschaft leicht vergessen lässt, welch wichtige Rolle er vorher schon auf allen Seiten des politischen Spektrums spielte. ${ }^{16}$ Ein weiterer Aspekt der politischen Kultur ist ihre visuelle Ausprägung, sind also die Bilder und Symbole, mit denen die Republik wie ihre Feinde sich selbst darstellten und die sich insbesondere auf den Wahlplakaten der Parteien fanden sowie bei Feiern und Aufmärschen gezeigt wurden. Das verweist schon auf den dritten wichtigen Aspekt, die symbolischen Praktiken, mit denen sich Parteien und Bewegungen vor allem im öffentlichen Raum präsentierten. $\mathrm{Zu}$ fragen ist somit neben der jeweiligen Selbstdarstellung insbesondere auch danach, wie mit dem politischen Gegner umgegangen wurde, im geschlossenen Raum des Parlaments und im öffentlichen Raum der Straße. Mit welchen Mitteln wurde hier Präsenz gezeigt, das eigene Programm vorgestellt, der eigene Machtanspruch verfochten? Welche Rolle spielte dabei physische Gewalt?

Hier können nun nicht alle aufgeführten Aspekte der politischen Kultur systematisch durchdiskutiert werden. Ausgehend vom Problem der Fragmentierung der politischen Kultur der Weimarer Republik und deren Zuspitzung zur Polarisierung ist vielmehr aufzuzeigen, was die Forschung mittlerweile über trennende, aber eben auch zusammenführende Elemente und Entwicklungen herausgearbeitet hat.

Hasso Hofmann, Legitimität gegen Legalität. Der Weg der politischen Philosophie Carl Schmitts, Berlin 21992, S. 66f.

15 Grundlegend zum Konzept „Politische Kultur“ und seiner Anwendung auf die Geschichte der Weimarer Republik: Detlef Lehnert / Klaus Megerle, Politische Teilkulturen zwischen Integration und Polarisierung. Zur politischen Kultur in der Weimarer Republik, Opladen 1990; Wolfgang Hardtwig (Hrsg.), Politische Kulturgeschichte der Zwischenkriegszeit 1918-1939, Göttingen 2005; ders. (Hrsg.), Ordnungen in der Krise. Zur politischen Kulturgeschichte Deutschlands 1900-1933, München 2007; Ute Daniel et al. (Hrsg.), Politische Kultur und Medienwirklichkeiten in den 1920er Jahren, München 2010.

16 Thomas Mergel, Führer, Volksgemeinschaft und Maschine. Politische Erwartungsstrukturen in der Weimarer Republik und im Nationalsozialismus, in: Hardtwig, Politische Kulturgeschichte (wie Anm. 15), S. 91-127, hier S. 97ff. 


\section{Gesellschaftliche Fragmentierung: „sozialmoralische Milieus“ und „Lager“}

Gewiss finden sich sehr deutliche Unterschiede der Lebenswelten in der Weimarer Republik - und damit sind die grundlegenden Einstellungen zur Politik angesprochen. Mit dem Begriff des „sozialmoralischen Milieus“, geprägt vom Soziologen M. Rainer Lepsius, hat die Forschung seit längerem versucht, die Selbst- und Fremdabgrenzung politisch überformter Lebenswelten zu beschreiben. ${ }^{17}$ Während des Kaiserreichs hatten sich in Deutschland vor allem zwei derartige Milieus herausgebildet: das katholische, politisch repräsentiert durch die Zentrumspartei, und das sozialistische, vertreten durch die Sozialdemokratie. Ein Netz von Vereinen, abgestimmt auf Altersstufen, Geschlechter und spezifische Zwecke wie den Sport, begleitete die Milieuangehörigen ,von der Wiege bis zur Bahre', sorgte für den Zusammenhalt und vermittelte ein klares Weltbild, angeleitet von den jeweiligen Deutungseliten. Innere Festigkeit und äußere Abschottung gingen Hand in Hand. Das mochte gerade bei einem Ortswechsel hilfreich sein. Beide Milieus konnten über Krieg und Revolution hinweg auch in den Weimarer Jahren ihre Bindekraft weitgehend erhalten, auch wenn sich ein kommunistisches Teilmilieu formte.

Weniger klar lagen die Dinge beim städtischen Bürgertum und bei der Landbevölkerung im protestantischen Deutschland. Vergleichbar enge Vereinsnetze fehlten hier, die lebensweltlichen Unterschiede waren erheblich, zwischen Großunternehmern und Gutsbesitzern auf der einen, Handwerksmeistern und Landarbeitern auf der anderen Seite, auch wenn auf dem Land patriarchalische Zuwendung gepaart mit sozialer Kontrolle für Zusammengehörigkeit sorgte, wie freiwillig oder erzwungen auch immer sie ausfiel. Krieg und Revolution sorgten hier für eine gewisse Auflockerung und Emanzipation der Landarbeiter, ohne dass sie sich allerdings in größerer Zahl dauerhaft an Parteien der Linken gebunden hätten. Ein oder mehrere Milieus mit derselben Kohärenz wie bei Katholiken und Sozialisten bildeten sich also nicht aus. Auf der Basis genauer Wahlanalysen, vor allem von Wählerwanderungen, hat der Politikwissenschaftler Karl Rohe deshalb vorgeschlagen, stattdessen von einem politischen „Lager“ zu sprechen, und zwar von einem „nationalen“, das er dem katholischen und sozialistischen Lager gegenüberstellt. Offenbar gelang es in den zwanziger Jahren der „Deutschnationalen Volkspartei“, der Nachfolgerin der Konservativen Partei des Kaiserreichs mit Basis auf dem protestantischen Land, auch städtische Wählerschichten zu erreichen. Damit zog sie Wählerstimmen zu sich herüber, die zuvor liberalen

17 M. Rainer Lepsius, Parteiensystem und Sozialstruktur. Zum Problem der Demokratisierung der deutschen Gesellschaft, in: ders., Demokratie in Deutschland. Demokratie in Deutschland. Soziologisch-historische Konstellationsanalysen. Ausgewählte Aufsätze, Göttingen 1993, S. 25-50. 
Parteien zugeflossen waren. Dies waren, als Nachfolger der beiden liberalen Parteien des Kaiserreichs, die anfänglich sehr starke linksliberale „Deutsche Demokratische Partei“, und die rechtsliberale „Deutsche Volkspartei“, bis zu seinem frühen Tod 1929 geführt von Gustav Stresemann. Am Ende stellte das „nationale Lager“ den größten Teil der NSDAP-Wählerschaft. ${ }^{18}$

Auch wenn dieses Lager nicht über ein gleich dichtes Vereinsnetz wie die anderen beiden verfügte, lässt sich doch seine organisatorische Verfestigung im Lauf der zwanziger Jahre erkennen. Eine wesentliche Kraft war dabei der „Stahlhelm. Bund der Frontsoldaten“, ein neuer Typus von Organisation, der nicht nur die Interessen von Kriegsveteranen vertreten wollte. ${ }^{19}$ Mit einer heroisierenden Kriegserinnerung verband er die Forderung nach einem dezidiert nationalen politischen Kurs und suchte dies durch Präsenz auf der Straße zu untermauern, etwa bei Veranstaltungen zum Reichsgründungstag. Neben dem immer weiter nach rechts rückenden „Stahlhelm“ spielten gerade in kleineren Orten auch die älteren Kriegervereine des Kyffhäuser-Bundes eine tragende Rolle. Um sie herum entwickelte sich in der lokalen Festkultur eine „militante Geselligkeit“, in der die Schützenvereine einen deutlichen Aufschwung nahmen, die Feuerwehren wuchsen und sich als eine Art Militärersatz definierten, ebenso wie die traditionsreichen Turnvereine. Diese bemühten sich, wie es etwa der Männerturnverein Celle bekundete, die durch den Wegfall der Wehrpflicht entstandene „Lücke in der körperlichen Erziehung der deutschen Jugend“ auszufüllen. Hier entstand jenseits der Zersplitterung der Parteienlandschaft eine Form bürgerlichprotestantischer Einigkeit, die nicht ausdrücklich gegen die Republik gerichtet war, aber mit der Ausgrenzung von Sozialdemokraten und auch Linksliberalen zumindest deutlich in diese Richtung wies. ${ }^{20}$

Zwischen den drei politischen Lagern bestanden somit relativ deutlich markierte subkulturelle Grenzen, die bei Wahlen kaum überschritten wurden, auch nicht zwischen 1930 und 1932, als die NSDAP zur größten Partei aufstieg, aber nur begrenzt Stimmen aus dem katholischen oder sozialistischen Lager, die Kommunisten eingeschlossen, sammeln konnte. ${ }^{21}$ Die neue Weimarer Massenkultur schliff die Grenzen zwischen den Milieus sowie Stadt und Land kaum ab, anders als lange angenommen. Das Radio war, angesichts der Empfangsmög-

18 Karl Rohe, Wahlen und Wählertraditionen in Deutschland. Kulturelle Grundlagen deutscher Parteien und Parteiensysteme im 19. und 20. Jahrhundert, Frankfurt 1992, S. 134-164.

19 Grundlegend weiterhin: Volker Berghahn, Der Stahlhelm. Bund der Frontsoldaten 1918-1935, Düsseldorf 1966.

20 Frank Bösch, Militante Geselligkeit. Formierungsformen der bürgerlichen Vereinswelt zwischen Revolution und Nationalsozialismus, in: Hardtwig, Politische Kulturgeschichte (wie Anm. 15), S. 151-182, das Zitat S. 162.

21 Jürgen W. Falter, Hitlers Wähler, München 1991, bes. S. 110-117. 
lichkeiten, hohen Gerätekosten und Gebühren und wegen seines bildungsorientierten Programms zur besten Abendsendezeit ein Medium vornehmlich des städtischen Bürgertums. Wer einen Film sehen wollte, tat dies - jedenfalls in der Stummfilmzeit - an sehr unterschiedlichen, wiederum vornehmlich städtischen Örtlichkeiten. Kinos für das Bürgertum orientierten sich am Theater; sie hielten eine Garderobe bereit, boten eine erlesene Musikbegleitung und erwarteten vornehmlich ruhigen Kunstgenuss. Kinos in Arbeitervierteln dagegen waren ein durchaus lauterer Ort, an dem das Publikum kam und ging, rauchte und trank, sich unbeschwerter äußerte und mit einfacherer Klavierbegleitung zufrieden sein musste. Das Filmangebot variierte stark, auch regional. Ein über alle Lagergrenzen hinweg bekannter und geliebter Filmstar wie Charlie Chaplin war die absolute Ausnahme, nicht die Regel. Auch in der jetzt aufblühenden Sportvereinswelt blieben die Lager- und Milieugrenzen grundsätzlich erhalten. ${ }^{22}$

Insgesamt zeichneten sich also die Milieus bzw. Lager als Träger der politischen Kultur durch große Kontinuität aus. Trotz - oder vielleicht gerade wegen - der großen Umbrüche der Kaiserzeit, dann des Ersten Weltkriegs, der Niederlage, Revolution und Inflation blieben sie erhalten. Eine gleichsam atomisierte Massengesellschaft entstand somit in der Weimarer Republik gerade nicht. Das bedeutet nun aber keineswegs zwingend eine unversöhnliche Polarisierung zwischen den Milieus und Lagern. Kooperation über ihre Grenzen hinweg war durchaus möglich. Die „Weimarer Koalition“, das Bündnis zwischen Sozialdemokraten, Zentrum und Linksliberalen, das die politische Ordnung der Weimarer Republik begründete, anfangs die Reichsregierung stellte und den größten Einzelstaat Preußen bis zum Vorabend der NS-Machtübernahme solide regierte, zeigt dies sehr deutlich. Ein weiterer Beleg ist die Regierungsbeteiligung der Deutschen Volkspartei unter ihrem Vorsitzenden und langjährigen Außenminister Gustav Stresemann. Auf die fragile Annäherung der Deutschnationalen Volkspartei an die Republik wird noch einzugehen sein. Milieubindung und pragmatische Regierungszusammenarbeit schlossen sich also keineswegs aus, auch wenn die jeweilige Parteibasis weniger beweglich war und ihre Führung bisweilen unter sehr starken Druck setzte.

22 Ross, Media (wie Anm. 8), S. 128-140, 156-174; Karl Christian Führer, Auf dem Weg zur ,Massenkultur'? Kino und Rundfunk in der Weimarer Republik, in: Historische Zeitschrift 262 (1996), S. 739-781; Stefan Nielsen, Sport und Großstadt 1870 bis 1930. Komparative Studien zur Entstehung bürgerlicher Freizeitkultur, Frankfurt 2002, S. 375ff., 431-433; Wilhelm Leo Guttsman, Workers' Culture in Weimar Germany. Between Tradition and Commitment, New York 1990, S. $134 \mathrm{ff}$. 


\section{Stabilisierungsfaktoren der Republik}

Ein festgefügtes „republikanisches Lager“ kann man somit nicht ohne weiteres ausmachen, wenn man danach fragt, wer die Republik trug und wie sich dies in der politischen Kultur niederschlug. Genauso falsch wäre es aber auch, wie dies

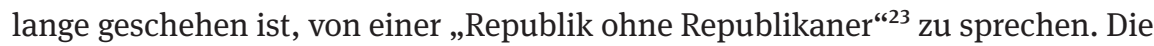
Republik hatte durchaus ihre - nicht wenigen - Verteidiger, und sie bemühte sich um den Aufbau einer eigenen Symbolwelt, an der auch die Bundesrepublik heute noch partizipiert, ohne dass dies allgemein bekannt ist.

Um mit dem letzten Punkt zu beginnen: Für die Gestaltung dieser Symbolwelt wurde eigens das Amt des „Reichskunstwarts“ geschaffen und dem aus Weimar stammenden Kunsthistoriker Edwin Redslob übertragen, der es bis 1933 innehatte. ${ }^{24}$ Redslob wollte Staatssymbole in „modernem Stil“. Zwei Ergebnisse dieser Bemühungen waren das Staatssiegel der Weimarer Republik sowie die Standarte des Reichspräsidenten, die beide später von der Bundesrepublik übernommen wurden und bis heute als Bundessiegel bzw. als Standarte des Bundespräsidenten in Gebrauch sind. ${ }^{25}$

23 Die Formulierung findet sich zeitgenössisch zunächst bei Ernst Troeltsch, „Aristokratie“ in: Der Kunstwart und Kulturwart: Monatsschau für Ausdruckskultur auf allen Lebensgebieten 33 (1919), S. 49-57, hier S. 50f. Troeltsch verweist allerdings auf diese „Rede [....], die man so oft hören kann“ (ebd., S. 50), um sich von ihr zu distanzieren; er sieht die mit der Republikgründung erreichte Demokratisierung als „unabänderlich“ (ebd.) an, ungeachtet seiner Forderung, klare politische Führung und die Ausbildung einer Leistungselite zu gewährleisten.

24 Christian Welzbacher (Hrsg.), Der Reichskunstwart. Kulturpolitik und Staatsinszenierung in der Weimarer Republik 1918-1933, Weimar 2010. Nach dem Zweiten Weltkrieg wirkte Redslob als Mitgründer und erster Rektor der Freien Universität Berlin.

25 Reichskunstwart Dr. Edwin Redslob, Die künstlerische Formgebung des Reichs, Berlin 1926, bes. S. 7-9; zur Übernahme der Symbole durch die Bundesrepublik 1949/50, die nicht als direkte Berufung auf das demokratische Erbe Weimars zu verstehen war, sondern den Zweck verfolgte, den westdeutschen Staat im Kontrast zur DDR als allein legitimierten Wahrer der nationalstaatlichen Tradition erscheinen zu lassen: Ullrich, Weimar-Komplex (wie Anm. 1), S. 471-487. 


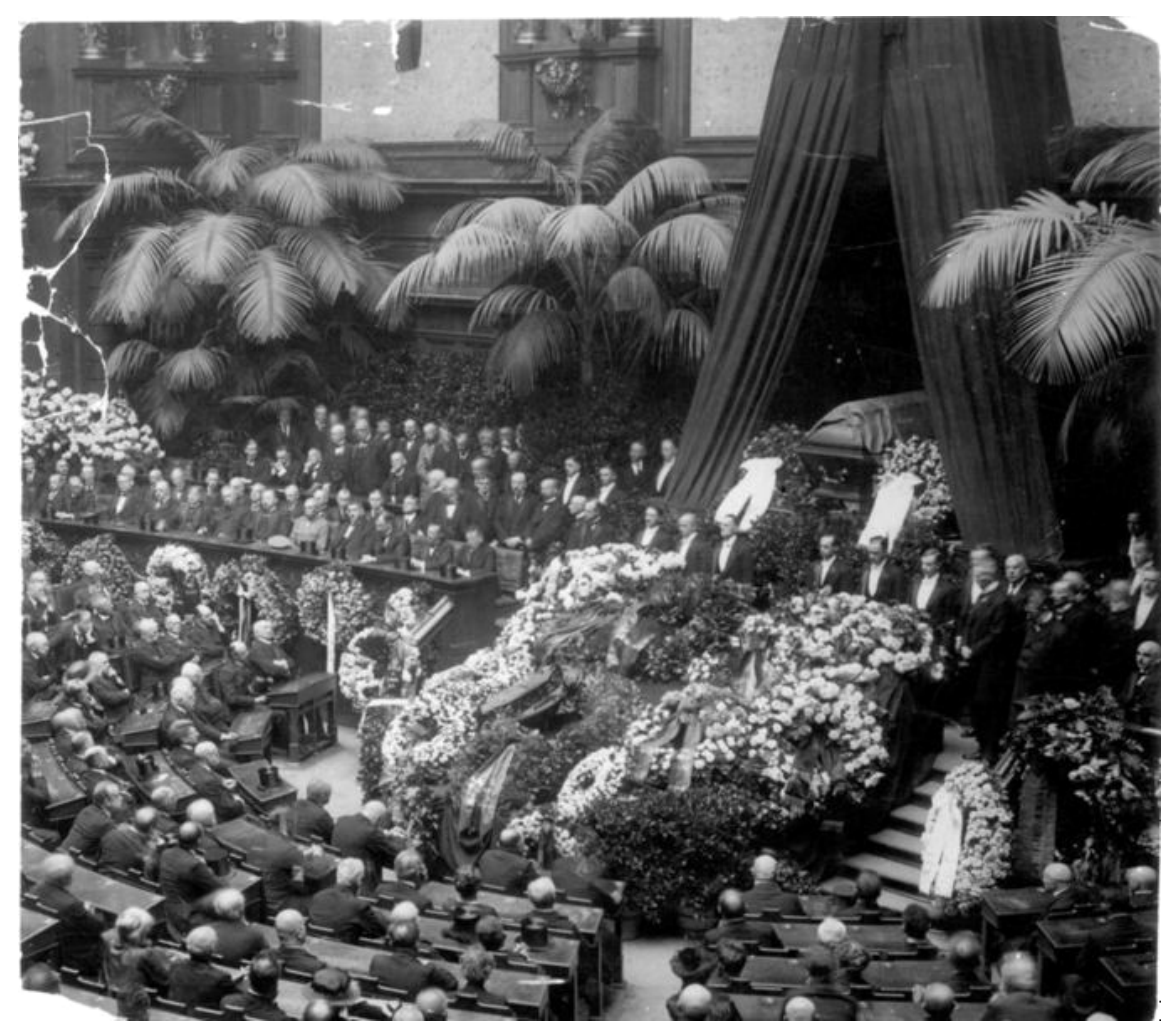

Abb. 1: Staatsakt für Walter Rathenau, Aufbahrung des Sarges im Reichstag am 27. Juni 1922. (Bundesarchiv, Bild 183-Z1117-502 / CC-BY-SA 3.0, CC BY-SA 3.0 de, https:// commons.wikimedia.org/w/index.php?curid=5438077)

Der Adler blieb also das Staatssymbol, aber er wurde jetzt klarer, prägnanter in der Form, ohne sich in völliger Abstraktion zu verlieren, eine Inkarnation sozusagen des Kompromisscharakters der Republik. Redslob gestaltete unter anderem auch die sehr eindringliche, monarchische Tradition gleichsam überschreibende Trauerfeier für den ermordeten Reichsaußenminister Walther Rathenau im Reichstag, in der Rathenaus Mutter in der vormaligen Kaiserloge Platz nahm und Wagners Trauermusik zu Siegfrieds Tod aus der Götterdämmerung die Dolchstoßhetze der extremen Rechten gegen sie selbst wandte. ${ }^{26}$

26 Manuela Achilles, Reforming the Reich: Democratic Symbols and Rituals in the Weimar Republic, in: Kerstin Barndt / Kathleen Canning (Hrsg.), Weimar Publics/Weimar Subjects: Rethinking the Political Culture of Germany in the 1920s, New York 2010, S. 175-191, hier S. 184-188. 
Republikanische Symbolpolitik war aber nicht allein Sache eines hohen Beamten. Reichspräsident Friedrich Ebert selbst wirkte hier als treibende Kraft, gerade nach dem Rathenau-Mord im Juni 1922, der die Republikaner keineswegs einschüchterte, sondern in ihrer Entschlossenheit zur Republikverteidigung bestärkte. Ebert drang auf die Abhaltung einer sichtbaren Verfassungsfeier am 11. August dieses Jahres mit abendlicher Festveranstaltung und anschließendem Fackelzug, den er selbst abnahm und bei dem er eine Rede hielt. Die Feier sollte nun zu einem jährlichen Ereignis werden. Außerdem machte er den Anfang der dritten Strophe des Deutschlandlieds zum Motto der Feier 1922 und erklärte sie dann in einem Erlass für die Reichswehr zur Nationalhymne. Zu einer eindringlichen Bekundung republikanischer Selbstbehauptung wurde die von ihm mitbetriebene Feier zum 75. Jahrestag der Revolution von 1848 in Frankfurt. Großen Beifall empfing Ebert, als er am Abend zu einer Aufführung von Beethovens Fidelio erschien; es folgte ein Fackelzug von 60-70.000 Teilnehmern, vor denen er in einer Rede die Leitgedanken der Revolution von 1848 - Einheit, Freiheit, Vaterland - auch zu denen der neuen Republik erklärte. Messbar war die Wirkung solcher Initiativen und Feiern nicht. Aber auch politische Gegner bescheinigten Ebert ein angemessenes, würdevolles Auftreten. ${ }^{27}$ Andere freilich überzogen ihn mit Hohn und Spott, etwa mit Hilfe des berühmt-berüchtigten Badehosenbildes von 1919. ${ }^{28}$ Doch es wäre einseitig, nur solche hasserfüllten Angriffe hervorzuheben und die Ansätze einer genuinen republikanischen Repräsentationskultur bei Ebert und unter seiner Ägide zu übersehen.

Noch mehr in die Breite wirkte eine Organisation, die die Farben der Republik in ihrem Namen führte: das „Reichsbanner Schwarz-Rot-Gold. Bund Deutscher Kriegsteilnehmer und Republikaner“. Gegründet 1924 in Reaktion auf den Hitlerputsch und das Anwachsen des bereits erwähnten „Stahlhelm“ zählte das Reichsbanner eine Million Mitglieder, deutlich mehr als der rechtsnationale Veteranenverband..$^{29}$ Die allermeisten waren Sozialdemokraten, beteiligt waren aber auch Angehörige des linken Zentrumflügels und der Linksliberalen, also der anderen Parteien der „Weimarer Koalition“, die im Vorstand des Verbandes prominent vertreten waren. In Aufmärschen und Kundgebungen zeigte das Reichsbanner öffentliche Präsenz, gerade zum Verfassungstag. So zogen am 11. August

$27 \mathrm{Zu}$ Eberts republikanischer Symbolpolitik: Walter Mühlhausen, Friedrich Ebert 1871-1925. Reichspräsident der Weimarer Republik, Bonn 2006, S. 816-848.

28 Walter Mühlhausen, Die Weimarer Republik entblößt. Das Badehosen-Foto von Friedrich Ebert und Gustav Noske, in: Gerhard Paul (Hrsg.), Das Jahrhundert der Bilder. 1900 bis 1949, Göttingen 2009, S. 236-243.

29 Grundlegend zum Reichsbanner: Karl Rohe, Das Reichsbanner Schwarz Rot Gold, Düsseldorf 1966; jetzt Benjamin Ziemann, Contested Commemorations. Republican War Veterans and Weimar Political Culture, Cambridge 2013, zur Problematik der Mitgliederzahlen S. 64-68. 
1929 in Berlin 75.000 Reichsbannerangehörige in einer eindrucksvollen, vier Stunden dauernden Parade vom Hohenzollernschloss über die Allee Unter den Linden zum Brandenburger Tor. Eine Massenveranstaltung im Stadion räumte der Jugend einen besonderen Platz ein. In anderen Jahren fanden solche zentralen Feiern etwa in Weimar, Nürnberg, Leipzig oder Koblenz statt.

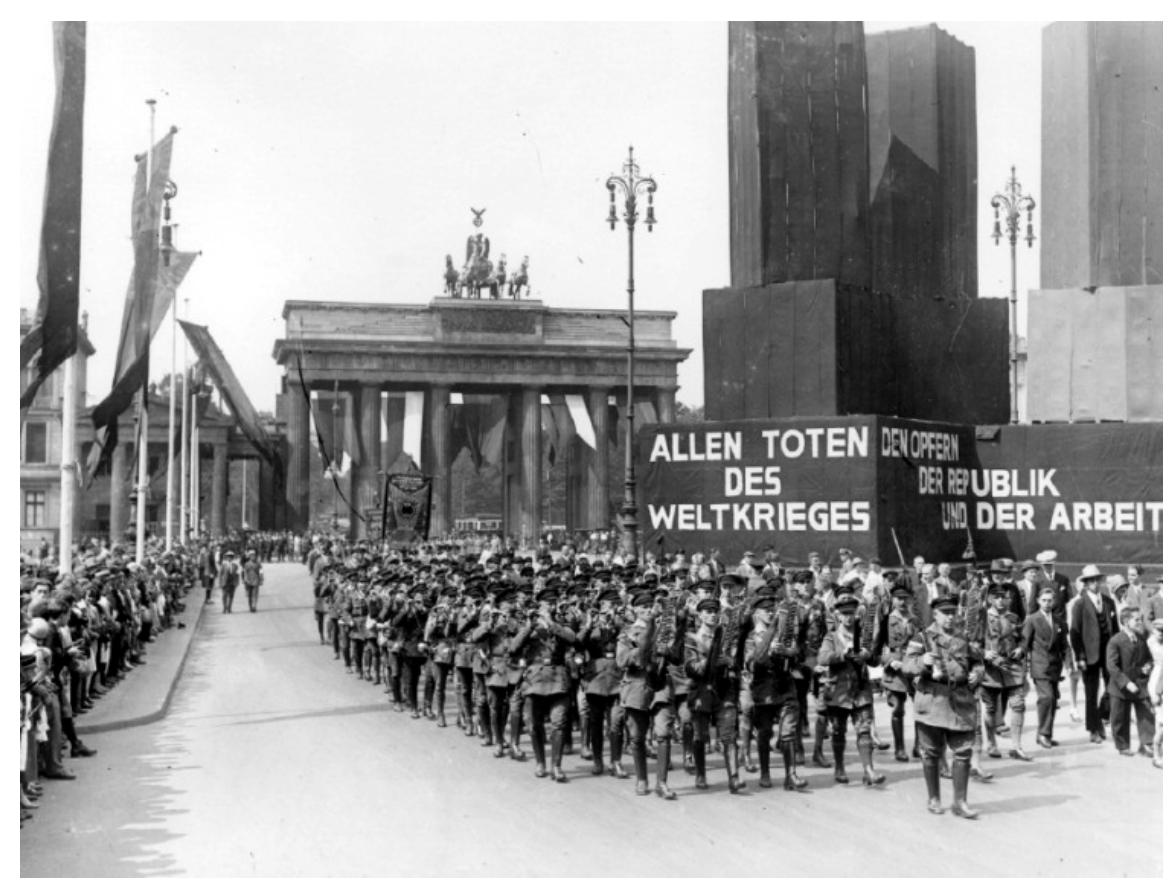

Abb. 2: Verfassungsfeier in Berlin am 11. August 1929, Vorbeimarsch des Reichsbanners am Brandenburger Tor. (https://commons.wikimedia.org/wiki/Category:Reichsbanner_SchwarzRot-old?uselang=de\#/media/File:Bundesarchiv_Bild_102-08216,_Berlin,_Reichsbanner_vor_ dem_Brandenburger_Tor.jpg)

Aber auch an vielen anderen Orten auf regionaler Ebene organisierte das Reichsbanner aus Anlass des Verfassungstags vergleichbare Veranstaltungen. Gerade bei diesen Feiern propagierte das Reichsbanner die Idee der „Volksgemeinschaft“ und besetzte damit einen wichtigen Begriff der politischen Sprache mit sozialreformerisch-inklusiver Stoßrichtung gegen eine ausgrenzende Definition auf der radikalen Rechten. ${ }^{30}$

30 Nadine Rossol, Performing the Nation in Interwar Germany. Sports, Spectacle and Political Symbolism, 1926-1936, Basingstoke 2010, bes. S. 61ff.; zur unterschiedlichen Akzeptanz des 
Das Reichsbanner war darüber hinaus ein Forum republikanischer Kriegserinnerung. Schon der „Reichsbund der Kriegsbeschädigten“, der mit 600.000 Mitgliedern größte und den Sozialdemokraten nahestehende Verband der Kriegsversehrten hatte seit Beginn der zwanziger Jahre eine glorifizierende Sicht des Krieges zurückgewiesen, die Leiden der Soldaten betont und in der alliierten Übermacht den Grund für die deutsche Niederlage erkannt. Damit wandte er sich scharf gegen die „Dolchstoß“-These der radikalen Rechten. Das Reichsbanner nahm diese Deutung auf und setzte sich im Verbund mit der französischen „Union Fédérale“ für internationale Verständigung ein. ${ }^{31}$ Die aktiven Republikaner waren also durchaus zahlreich und sie waren in der Öffentlichkeit präsent.

Deutlicher als früher wird mittlerweile auch der Anteil der Unterstützer der Republik an den intellektuellen Debatten der Zeit über die richtige politische Ordnung gesehen. Auch solche Debatten tragen zur Ausgestaltung der politischen Kultur bei. Lange Zeit hat sich die Aufmerksamkeit auf das plakativere „antidemokratische Denken“ konzentriert, vor allem am rechten Rand des politischen Spektrums. Doch es waren nicht nur einige wenige prominente Künstler und Intellektuelle wie Thomas Mann, die sich für die Republik einsetzten. Heute in der Öffentlichkeit weitgehend vergessene Staatsrechtler wie Gerhard Anschütz oder Hermann Heller stellten sich dezidiert auf den Boden der demokratischen Verfassung und suchten sie weiter auszugestalten. Dass dem Parlament die zentrale Rolle in der Republik zukam, stand für sie außer Frage, ebenso die Überlegenheit des 1919 eingeführten Verhältniswahlrechts. Denn für sie war das Volk keine homogene Einheit mit vermeintlich einheitlichem Willen, der in punktuellen Abstimmungen bloß erfragt oder von einem wie auch immer gearteten „Führer“ erspürt werden konnte. Sie erkannten die Pluralität der modernen Gesellschaft und suchten ihr Rechnung zu tragen. Dabei waren sie durchaus nicht einheitlicher Auffassung darüber, wie groß etwa der Einfluss von Interessenverbänden im Verhältnis zu dem der Länder- und Kommunalvertretungen sein sollte, in welchem Maß auf die öffentliche Meinung und historische Traditionen bei der Gesetzgebung Rücksicht zu nehmen oder welcher Umfang des Sozialstaats angemessen war. Nach dem Aufschwung der radikalen Parteien, besonders der NSDAP, seit 1930 liebäugelten sie zeitweise mit dem Gedanken einer starken Exekutive, die die radikalen Kräfte in Schach halten sollte, verfochten aber eine unbedingte Bindung der Politik an das Recht und verwarfen jegliches Kokettie-

Volksgemeinschaftskonzepts in der Organisation Ziemann, Commemorations (wie Anm. 29), S. 125-127.

31 Ziemann, Commemorations (wie Anm. 29), S. 128-162, zu dem beim Aufmarsch 1929 gezeigten temporären Ehrenmal für die Toten des Krieges und den Opfern die für die Republik S. 188-191. 
ren mit dem Ausnahmezustand..$^{32}$ Das Tastende, Suchende der Zeit und gewisse mentale Überhänge des Kaiserreichs - der ,starke Staat ‘ - sind hier erkennbar, genauso aber der klare Wille, die Fundamente einer demokratischen Republik festigen zu helfen.

Zählt man zu diesen entschiedenen Anhängern der Republik weitere „Vernunftrepublikaner“, die sich aus pragmatischen Gründen auf den Boden der neuen demokratischen Ordnung stellten, im Kreis der Staatsrechtslehrer wie anderer Universitätsprofessoren, aber auch in der Wirtschaft, so wird sichtbar, dass die Republik auch unter den Eliten auf einen durchaus nicht kleinen Unterstützerkreis, wiewohl unterschiedlicher Nachdrücklichkeit, zählen konnte. ${ }^{33}$

Auch der zentrale Ort, an dem die Meinungskämpfe auf nationaler Ebene ausgetragen wurden, der Reichstag, erscheint heute in etwas anderem Licht, als dies lange Zeit der Fall war. Die Instabilität der Regierungen und die Obstruktionshaltung der extremen Parteien haben hier das Bild bestimmt. Mittlerweile wird aber auch die integrative Seite des Reichstags betont. Wie der Berliner Historiker Thomas Mergel gezeigt hat, wuchs mit dem Bedeutungsgewinn des Parlaments in der neuen Republik das Gewicht seiner Ausschüsse, in denen die eigentliche Sacharbeit geleistet wurde. Hier waren Grundsatzfragen ausgeklammert, es dominierte ein Ton der Höflichkeit und Sachlichkeit, enge Beziehungen von Experten bildeten sich über Parteigrenzen hinweg. In Krisensituationen half die „Sprache des Staatsmanns“ schwierige Entscheidungen gegenüber der eigenen Klientel zu rechtfertigen und Gemeinsamkeiten zu stiften. So rückten im Lauf der zwanziger Jahre die Parteien näher aneinander, sogar unter Einschluss einzelner Kommunisten und Nationalsozialisten. Eine gewisse Annäherung zeigte besonders die Deutschnationale Volkspartei, deren Basis der Republik zwar ablehnend gegenüberstand, deren Führung im Reichstag aber sich zur konstruktiven Mitarbeit bereitfand, dem „integrativen Sog“ der Parlamentsarbeit folgend und beeinflusst auch noch von ihrer traditionellen Orientierung auf ,den Staat ' hin. ${ }^{34}$ Wie weit diese „stille Republikanisierung“ der Deutschnationalen tatsächlich reichte, ob sie sich weiter verfestigt hätte, wenn der Republik mehr Zeit geblieben wäre, ist in der Forschung umstritten. ${ }^{35}$ Aber dass sich für die bis zum Aufstieg

32 Groh, Staatsrechtslehrer (wie Anm. 13), bes. S. 579-586.

33 Andreas Wirsching / Jürgen Eder (Hrsg.), Vernunftrepublikanismus in der Weimarer Republik. Politik, Literatur, Wissenschaft, Stuttgart 2008.

34 Thomas Mergel, Parlamentarische Kultur in der Weimarer Republik. Politische Kommunikation, symbolische Politik und Öffentlichkeit im Reichstag, Düsseldorf 2002, die Zitate S. 228, 277. 35 Mergel, Parlamentarische Kultur (wie Anm. 34), S. 323; die Gegenposition: Manfred Kittel, „Steigbügelhalter“ Hitlers oder „Stille Republikaner“? Die Deutschnationalen in neuerer politikgeschichtlicher und kulturalistischer Perspektive, in: Hans-Christof Kraus / Thomas Nicklas (Hrsg.), Geschichte der Politik. Alte und Neue Wege, München 2007, S. 200-235; näher an Mergel: 
der NSDAP 1930 größte Partei im „nationalen Lager“ eine Perspektive innerhalb der Republik eröffnete und ihre Mitarbeit nicht allein mit taktischen Gründen zu erklären ist, das steht inzwischen außer Frage.

\section{Belastungen}

Der genauere Blick auf Sprache und Umgangsformen im Reichstag zeigt allerdings auch ein grundsätzliches Problem auf. Hinter der Sprache der Sachlichkeit trat zurück, dass es immer auch um spezifische, von entsprechenden Organisationen artikulierte Interessen ging. Eine angemessene Sprache für diesen Interessenkampf vermochte das Parlament jedoch nicht zu entwickeln. Er fand in Hinterzimmern statt und wurde auf Wahlplakaten sichtbar, aber nicht in den Ausschüssen und auch nicht in den Plenumsdebatten, in denen zwar Grundsatztreue und Glaubwürdigkeit im Vordergrund standen, aber doch so, dass die etablierten Arbeitsbeziehungen zu den anderen Parteien nicht beschädigt wurden. Die mediale Öffentlichkeit jedoch nahm den Interessenkampf wahr und stellte ihm das Ideal einer vermeintlich harmonischen „Volksgemeinschaft“ entgegen, in der alle Interessen gleichermaßen berücksichtigt werden sollten. Einem solchen Ideal konnte der Reichstag freilich nicht genügen, auch nicht der widersprüchlichen Erwartung, dass er auf der einen Seite das Volk möglichst genau abbilden, auf der anderen aber „Führer“ hervorbringen sollte, die gerade unabhängig von spezifischen Interessen und Bindungen agieren würden. So war der Reichstag lange Zeit eine durchaus integrierende Institution, aber er stand dabei unter dem überhöhten Erwartungsdruck einer gespaltenen Gesellschaft. ${ }^{36}$

Die sich aus dieser Fragmentierung der Gesellschaft ergebende Polarisierung entlang der Trennlinien politischer Milieus und Lager bildete einen grundlegenden Belastungsfaktor der Republik. Wenn man nun daneben spezifische Zuspitzungen insbesondere in der zweiten Hälfte der zwanziger Jahre ins Auge fasst, dann wird wiederum erkennbar, wie sehr die Weimarer Republik Nachgeschichte eines großen und verlorenen Krieges war. Dies meint vornehmlich die Entwicklung der politisch motivierten Gewalt, allerdings in weniger direkter Form, als es zunächst den Anschein haben mag. Der Bürgerkrieg der ersten Jahre war punktuell - erst danach machten die „Wehrverbände“ der politischen Lager, die ja zunächst einmal Veteranenverbände waren, Gewalt zu einem an vielen Orten erfahrbaren Phänomen. Allerdings entfalteten sie sich erst seit 1924. Der „Stahl-

Maik Ohnezeit, Zwischen „schärfster Opposition“ und dem „Willen zur Macht“. Die Deutschnationale Volkspartei (DNVP) in der Weimarer Republik 1918-1928, Düsseldorf 2011.

36 Mergel, Parlamentarische Kultur (wie Anm. 34), S. 362ff. 
helm“ und der kleinere „Werwolf“ auf der Rechten, der zunächst hier einzuordnende, später in die Mitte rückende „Jungdeutsche Orden“, das „Reichsbanner“ und der „Rote Frontkämpferbund“ der Kommunisten sowie dann die nationalsozialistische SA (die sich allerdings nicht als Veteranenverband, sondern als Formation junger Kämpfer verstand) stritten um die Kontrolle des öffentlichen Raums, über die Jahre an Mitgliedern und Ortsgruppen zunehmend und mit wachsender Zahl der Opfer, die im blutigen, von mehreren großen Wahlgängen gekennzeichneten Jahr 1932 kulminierte. ${ }^{37}$

Dies belastete ganz gewiss die politische Kultur - allerdings muss sehr deutlich unterstrichen werden, dass das Ausmaß der Gewalt, gemessen an der Zahl der Todesopfer, auch nach 1930 weit hinter dem zurückblieb, was in den Bürgerkriegsjahren 1919 und 1920 zu verzeichnen gewesen war. ${ }^{38}$ Ein zur Selbstverteidigung entschlossener demokratischer Staat hätte die Gewalt auch 1932 eindämmen und kontrollieren können, aber das war er auf Reichsebene seit dem Amtsantritt des nur noch vom Reichspräsidenten gestützten Kabinett Papen im Mai 1932 nicht mehr. Das Problem war also nicht die Gewalt an sich, sondern ihre Instrumentalisierung, vor allem durch jene Kreise auf der extremen Rechten, die entschlossen waren, die Republik zu beseitigen, alle Schuld an der Gewalt den Kommunisten und Sozialdemokraten in die Schuhe schoben und die der eigenen Seite als berechtige Gegenwehr darstellten. Dies verband sich mit einer Kriegserinnerung, in der nun in der Öffentlichkeit die heroische Selbstsicht der Veteranen die Oberhand gewann. Sie erschien mittlerweile offenbar auch jungen Menschen auf der Linken attraktiver als die auf Leid und sinnloses Opfer fokussierte der älteren republikanischen Veteranen. ${ }^{39}$

Ohne die Weltwirtschaftskrise wären solche Zuspitzungen wohl nicht ganz vermieden worden, aber weniger gravierend ausgefallen. Doch der Börsensturz des „schwarzen Freitag“ 1929 und seine katastrophischen Folgen in den kommenden Jahren verschafften all denjenigen massiven Auftrieb, die nur auf die richtige Gelegenheit warteten, der Republik den Todesstoß zu versetzen. Gerade sie priesen eine harte, unzweideutig auf Sieg setzende Männlichkeit als Kardinaltugend, gegen alles ,Weichliche“, Verhandlung und Kompromiss betonende,

37 Dirk Schumann, Politische Gewalt in der Weimarer Republik 1918-1933. Kampf um die Straße und Furcht vor dem Bürgerkrieg, Essen 2001.

38 Dirk Schumann, Gewalterfahrungen und ihre nicht zwangsläufigen Folgen. Der Erste Weltkrieg in der Gewaltgeschichte des 20. Jahrhunderts, in: Zeitgeschichte-online, Thema: Fronterlebnis und Nachkriegsordnung. Wirkung und Wahrnehmung des Ersten Weltkriegs, Mai 2004, URL: <http://www.zeitgeschichte-online.de/thema/gewalterfahrungen-und-ihre-nicht-zwangslaeufigen-folgen>, S. 14 (PDF).

39 Schumann, Politische Gewalt (wie Anm. 37), S. 338ff.; Arndt Weinrich, Der Weltkrieg als Erzieher. Jugend zwischen Weimarer Republik und Nationalsozialismus, Essen 2011, S. 108-111. 
das dabei auch häufig als ,jüdisch“ verurteilt wurde. Für den „kraftvollen Mann“ Adolf Hitler, wie er sich in einer Rede 1928 anpries, waren solche Formeln rhetorischer Standard..$^{40}$

\section{Gegenwartsbezüge}

Was lässt sich nun möglicherweise aus der Geschichte der politischen Kultur in der Weimarer Republik für heute lernen? Wie fern Weimar der Gegenwart zunächst einmal ist, sollte deutlich geworden sein: Weder die fundamentalen Umbrüche zur Moderne noch die Folgen des ersten die ganze Gesellschaft erfassenden Krieges bedürfen heute einer Auseinandersetzung. Die Deutschen haben nach 1945 in vielen Jahrzehnten Demokratie gelernt, seit Ende der fünfziger Jahre unter relativ komfortablen, weitere Zuwächse versprechenden materiellen Verhältnissen und in einer klaren Weltordnung, die - jedenfalls in Europa - den Frieden sicherte. Diese Sicherheiten sind heute allerdings verlorengegangen, wenn auch bisweilen mehr in der Wahrnehmung als in der Realität.

Die neue Zukunftsunsicherheit macht nun sehr alte politische Formeln für manche wieder attraktiv, in Deutschland wie auch in anderen Teilen Europas und jenseits des Atlantiks. An diesem Punkt lassen sich dann eben doch gewisse Ähnlichkeiten mit der politischen Sprache der rechtsradikalen Feinde der Weimarer Republik erkennen. Das vermeintlich homogene Volk und der vermeintlich ,starke Mann', der es ,versteht‘ und führt, scheinen Halt und einen Heilsweg aus der unübersichtlichen Gegenwart zu versprechen. Dem lässt sich wiederum - damals wie heute - die Gemeinsamkeit der Demokraten entgegenstellen, die wissen, dass die moderne Gesellschaft eben doch viel komplizierter und vielfältiger ist und dass auftrumpfendes Gehabe nicht mit politischer Führung verwechselt werden darf. Die starke Zivilgesellschaft, die sich in den vergangenen Jahrzehnten in Deutschland entwickelt hat, muss dabei freilich, anders als in Weimar, nicht auf militärähnliche Verhaltensmuster zurückgreifen, um sich solchen Vereinfachungen entgegenzustellen. Und der demokratische Rechtsstaat hat ausreichende Mittel, Gewalt zu unterbinden; er muss sie nur entschlossen einsetzen, wie dies am Ende der Weimarer Republik nicht mehr der Fall war.

Verzagtheit ist also nicht angemessen, wenn die Chancen zum Erhalt der Demokratie erwogen werden. Freilich wäre genauer zu überlegen, wie sich präziser als in der Weimarer Republik Interessen und Bedürfnisse benennen lassen,

40 Martina Kessel, Demokratie als Grenzverletzung. Geschlecht als symbolisches System in der Weimarer Republik, in: Gabriele Metzler / Dirk Schumann (Hrsg.), Geschlechter(un)ordnung und Politik in der Weimarer Republik, Bonn 2016, S. 81-108, das Zitat S. 102. 
vor allem auch derer, die sich ,abgehängt‘ fühlen. Dies zu erreichen und daraus zivilisierte Formen der Kontroverse zu entwickeln, sollte eine lohnende Aufgabe sein. Die beschleunigte Medienwelt von heute macht das auf ihre Weise ähnlich schwierig wie der hohe Erwartungsdruck in der Weimarer Republik. Aber eine Alternative gibt es nicht. Politik bleibt ein mühsames Geschäft, „ein starkes langsames Bohren von harten Brettern mit Leidenschaft und Augenmaß zugleich“, wie dies Max Weber in seinem berühmten Vortrag „Politik als Beruf“ 1919 formuliert hat. ${ }^{41}$ Es gilt also, der Hohlheit selbsternannter Volksversteher auf beiden Seiten des Atlantiks den Charme von Sachkunde, Geduld und Kompromissfähigkeit entgegenzustellen, mit Nachdruck und Entschiedenheit.

41 Max Weber, Politik als Beruf (1919), in: Wolfgang J. Mommsen / Wolfgang Schluchter in Zusammenarbeit mit Birgitt Morgenbrod (Hrsg.), Max Weber, Wissenschaft als Beruf (1917/1919), Politik als Beruf (1919) (= Max Weber Gesamtausgabe, Abt. I, Bd. 17), Tübingen 1992, S. 156-252, das Zitat S. 251f. 